\title{
Health professional education must connect science to society
}

\author{
K. Srinath Reddy \\ President, Public Health Foundation of India, New Delhi
}

Convocation address delivered on 25th October 2013, at Tirupati on the occasion of the 4th convocation of Sri Venkateswara Institute of Medical Sciences, Tirupati.

Srinath Reddy K. Health professional education must connect science to society. J Clin Sci Res 2014;3:51-4.

It is a proud privilege and a humbling honour for me to be here today to deliver the Convocation Address. While I have been well acquainted with the sterling reputation and remarkable record of SVIMS, it is with awe and admiration that I stand here today on my first visit to its campus. I feel specially blessed to be part of this sacred ceremony, where many young health professionals will be embarking on their professional careers after receiving their richly earned degreesin such a sanctified setting.

I would like to commence my address by congratulating all the graduates who are being recognized and rewarded for the successful completion of their scholastic journey. I would also like to express our collective appreciation and gratitude to the dedicated faculty who have devoted their time and talent to teach, train, mentor and motivate these graduates. Special thanks are due to the Director, Prof. Vengamma, who has provided inspirational leadership and exemplary commitment to excellence.

Occasions such as this call not only for a celebration of individual and institutional success, but also for a serious reflection on the evolution of medical education as it adapts to the changing context of the $21^{\text {st }}$ century. In the past decade there has been an extensive global debate on the purpose, process and products of health professional education, as health systems everywhere face new challenges and see new opportunities.

What is the purpose of medical education? Charles Mayo, one of the founders of the famed Mayo Clinic, said "There are two objects of medical education, to heal the sick and advance the science". That was in the late $19^{\text {th }}$ century. Now we have to add a third objective - to protect and promote the health of the population. The role of a health professional today is much more than that of the black bag carrying solo practitioner of the $19^{\text {th }}$ century, though the care, concern, compassion and courtesy that marked the professional ethic of those dedicated doctors is worth recalling and replicating in an era where the medical profession is in the danger of slipping in to a mercenary trade from its pedestal of a noble vocation. The role of a good health professional - especially those who work in institutions of higher, learning like SVIMS - is now much greater. You have to constantly relate your role to the larger social context.

Science and technology are powerful propellants of societal progress and are among the most visible hallmarks of advancing human civilization. However, science is sterile if it lacks social relevance and technology is a mere toy if it cannot transform social conditions. It

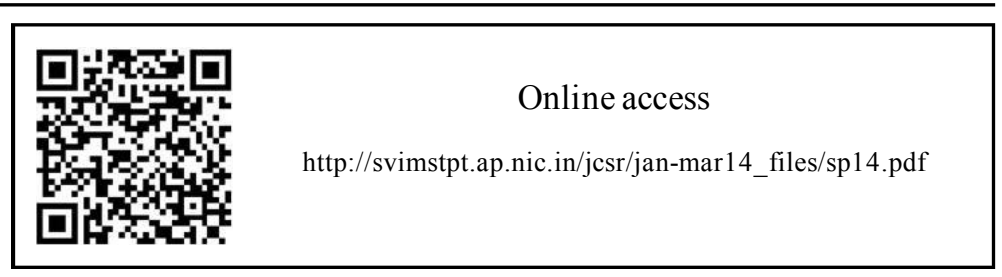


is, therefore, essential that we set the course of our professional lives on a path that promotes collective welfare and well-being while satisfying individual aspirations for a rewarding career. As advanced technologies increasingly dominate our professional practice, we need to ensure that the medicine does not become impersonal in its approach to individuals while all health professionals also work together to promote health in all its dimensions across society.

For this to happen, we need to adopt two mantras in health professional education and research: inter-disciplinarity and health system connectivity. This is clearly evident whether we look at basic sciences, clinical medicine or public health. Everywhere, there is need for breaking out of silos and building partnerships.

Basic research in life sciences needs a fusion of several disciplines, to discover the causes and cures of disease as well as developing the pathways for protecting and promoting health. These range from biotechnology to biomedical engineering and from nanophysics to computational chemistry and computational biology. The clinical disciplines too need to interact among themselves and integrate knowledge from other streams to enable appropriate and adequate clinical management. Clinical decision making too is no longer based only on knowledge of human biology and pathology. It is conditioned by inputs ranging from epidemiology to economics and is influenced by perspectives from ethics and patient rights.

The precept and practice of public health are even more broad based. In public health, the spectrum of research ranges from molecules to markets and its arena of action extends from reduction of risk factors to promotion of rights based approaches to universal and individual health. The platform of public health needs to integrate human biology with social and behavioural sciences, quantitative sciences like epidemiology, statistics and demography as well as economics and management sciences. It also has to partner with other educational streams to create conjoint programs. Public health engineering, public health law and environmental health are only a few examples of such trans-disciplinary collaborations.

Even within the conventional paradigm of health sciences, we have to ensure a seamless continuum between basic biomedical sciences, clinical medicine and public health. The scientific foundations of clinical diagnosis and care are provided by robust research in basic science. Public health too needs to be strengthened in design and delivery, to prevent avertable disease and also to create strong health systems which can ensure effective service delivery. Most of the burdens of infectious diseases and non-communicable diseases are eminently preventable. By strengthening our public health systems, we can avoid the overload of secondary and tertiary care services by diseases that could and should have been prevented in the first place or managed early at the primary care level. That way clinical medicine can also be practiced more efficiently, tending only to those who really need higher levels of care. The available resources can also be better shared by different clinical departments, since the overall patient load reaching hospitals would be far fewer if prevention and primary care function as effective filters. Clinicians who traditionally regard public health as a poor cousin should therefore recognize public health not only as a socially beneficial investment but also as a true ally of clinical medicine.

The spectrum of science is reductionist in content but holistic in context. This requires depth as well as breadth in knowledge generation and transformation of knowledge to wisdom, through integration and intelligent 
interpretation of information from multiple sources. No profession can exist in a silo and no academic discipline today can refuse to collaborate with another. Physical, biological and social sciences are constantly combining, to unravel the many mysteries of our existence and to solve the major problems that confront us in our daily lives. Words like health informatics, big data, neuro cybernetics, and Bayesian theorem of conditional probability are now part of the lexicon of medical research and health technologies. Whether it is organ imaging or m-health, development of drugs, devices and vaccines, gene therapy or robotic surgery, the value of inter-disciplinary collaboration is all too evident in the arena of health. People from diverse disciplines have now become cocreators of health. This institution too must reach out to develop fruitful partnerships with other institutions which have strengths in disciplines which currently do not exist within its precincts.

There is also a need for closer linkages between the education system and the health system, to closely align the settings, content and methodology of health professional education to the changing needs of health services and evolving architecture of the health system.

One of the major movements in global health, in recent years, has centered around the concept of Universal Health Coverage. The World Health Organization and the United Nations have endorsed it and have called upon all countries to re-configure their health systems to deliver UHC. This simply means that all persons must get all the health services they need without suffering financial hardship. These include promotive, preventive, curative and rehabilitative services, with a strong emphasis on effective and equitable primary health care. India too needs to move swiftly and surely in that direction. Nearly $70 \%$ of India's health care expenditure comes from private out of pocket spending. About 60 million Indians are pushed below the poverty line every year because of unaffordable health care costs.

I had the privilege of chairing the Planning Commission's High Level Expert Group on Universal Health Coverage (UHC). The $12^{\text {th }}$ Five Year Plan has adopted UHC as the key guiding principle. States like Karnataka and Kerala have announced UHC pilots in selected districts. I hope the Government of Andhra Pradesh will also implement all elements of UHC very soon. It is also necessary that health professionals also vigorously advocate for UHC, since that represents our profession's commitment to protect people's health. The $19^{\text {th }}$ century medical scientist and philosopher Rudolph Virchow said "physicians are the natural attorneys of the poor". We have to live up to that role by speaking up for the people and standing by the patient who needs essential health care. Without UHC, not only are patients denied needed care but doctors too cannot play a role which is true to their profession.

In a people centered health system, team work has become more essential than ever before. We may train as doctors, nurses, paramedics, technology developers or managers of complex systems. However, we need each other to get the best out of our own work and have to team up to collectively contribute to our maximum ability, as a group. All over the world, the roles of health professionals are being redefined and responsibilities are being reassigned, not only to overcome shortages of doctors and nurses but also to increase efficiency and decrease health care costs. Many functions are being transferred from specialist physicians to nonspecialist doctors, from doctors to specialist nurses and other mid-level health workers, from nurses to community health workers and so on. We too need to do that. This would require 
mutual respect among all categories of health professionals, researchers and managers and a non-hierarchical culture of teamwork. These do not emerge spontaneously in traditional educational establishments or old fashioned work environments. It is institutions such as yours that can act as crucibles for creating a culture of inter-disciplinarity and become cradles for nurturing inter-professional teams. I am sure this institute will create a new generation of innovative, adaptive, collaborative, skilled and socially responsible graduates who will act as effective agents of change, to transform our health system and enrich our society with their contributions.

As I conclude, I would like to wish the new graduates the very best of success and the highest level of fulfillment in their future careers. Convocations merely mark a milestone in your career. May the journey ahead be smooth and steady, as you move resolutely towards your goals. Even as you cross this threshold, you must resolve to remain lifelong learners, with unsated appetite for acquiring new knowledge and unabated energy for applying that knowledge to stimulate and steer desirable social change.

In your careers, you must seek a difference and make a difference! Be the master of your destiny but also be a servant of the society. Through our lives and work, each of us must aim to move the world to a better place. That is the purpose of individual lives in the long and uninterrupted story of human civilization which connects us to past and future generations. Whatever you do, make sure you leave your mark on the social progress of our country and make your alma mater proud that you were once in these portals as a student. Best of Luck!

Thank You For Your Attention. 